\title{
QCD factorization at forward rapidities
}

\author{
J. Nemchik \\ Czech Technical University in Prague, FNSPE, Břehová 7, 11519 Prague, Czech Republic \\ and \\ Institute of Experimental Physics SAS, Watsonova 47, 04001 Košice, Slovakia \\ E-mail: nemcik@saske.sk
}

\section{M. Šumbera*}

Nuclear Physics Institute AS CR, 25068 Řež/Prague, Czech Republic

E-mail: sumbera@ujf.cas.ca

We analyze several reactions on nuclear targets at forward rapidities and different energies (at smallest experimentally accessible Bjorken $x_{2}$ in the target). Nuclear effects are then usually interpreted as a result of shadowing or the Color Glass Condensate. QCD factorization of soft and hard interactions requires the nucleus to be an universal filter for different Fock components of the projectile hadron. We demonstrate, however, that this is not the case in the vicinity of the kinematic limit, Feynman $x_{F} \rightarrow 1$, where sharing of energy between the projectile constituents becomes an issue. The rise of suppression with $x_{F}$ is confirmed by the E772 and E886 data on the Drell-Yan and heavy quarkonium production. We show that this effect can be treated alternatively as an effective energy loss proportional to initial energy. This leads to a nuclear suppression at any energy, and predicts $x_{F}$ scaling of the suppression. We demonstrate also that the same kinematic limit can be approached in transverse momentum when the Cronin enhancement of particle production at medium-high $p_{T}$ switches to a suppression at larger $p_{T}$ violating thus QCD factorization. Such an unexpected effect seems to be confirmed by the RHIC data for pion production in $\mathrm{d}+\mathrm{A}$ collisions, and even for direct photons in Au+Au collisions.

35th International Conference on High Energy Physics - ICHEP 2010

July 22-28, 2010

Paris, France

${ }^{*}$ Speaker. 


\section{Introduction}

If a particle with mass $m$ and transverse momentum $p_{T}$ is produced in a hard reaction then the corresponding values of Bjorken variable in the beam and the target are $x_{1,2}=\sqrt{m^{2}+p_{T}^{2}} e^{ \pm y} / \sqrt{s}$. Thus, the forward rapidity region $(y>0)$ allows to study already at RHIC coherent phenomena (shadowing, Color Glass Condensate (CGC)), which are expected to suppress particle yields.

Interpretation of large- $y$ suppression at RHIC via coherent phenomena should be realized with a great caution since there is no consensus so far about the strength of gluon shadowing and CGC. The BRAHMS data [1] at $y=3.2$ to be explained are just fitted [2]. Besides, an energetic universality of a large- $y$ suppression is manifested so far for any reaction. Namely, all fixed target experiments have too low energy for the onset of coherence effects. The rise of suppression with $y$ (with Feynman $x_{F}$ ) shows the same pattern as that observed at RHIC. Such a feature common for all known reactions allows to favor another mechanism [3] which describes observed suppression via energy conservation effects in initial state parton rescatterings alternatively interpreted as a parton effective energy loss proportional to initial energy and leading to $x_{F}$ scaling of nuclear effects.

In the vicinity of the kinematic limit any hard reaction can be treated as a large rapidity gap (LRG) process where no particle is produced within rapidity interval $\Delta y=-\ln (1-x)$ ( $x$ coincides with $x_{1}$ or $x_{F}$ ). The suppression factor as a survival probability for LRG was estimated in [3], $S(x) \sim 1-x$. Each of multiple interactions of projectile partons produces an extra $S(x)$.

With such a suppression factor and applying the AGK cutting rules [ [] with the Glauber weight factors, we present in this paper a parameter-free description [], 5] of data for several reactions.
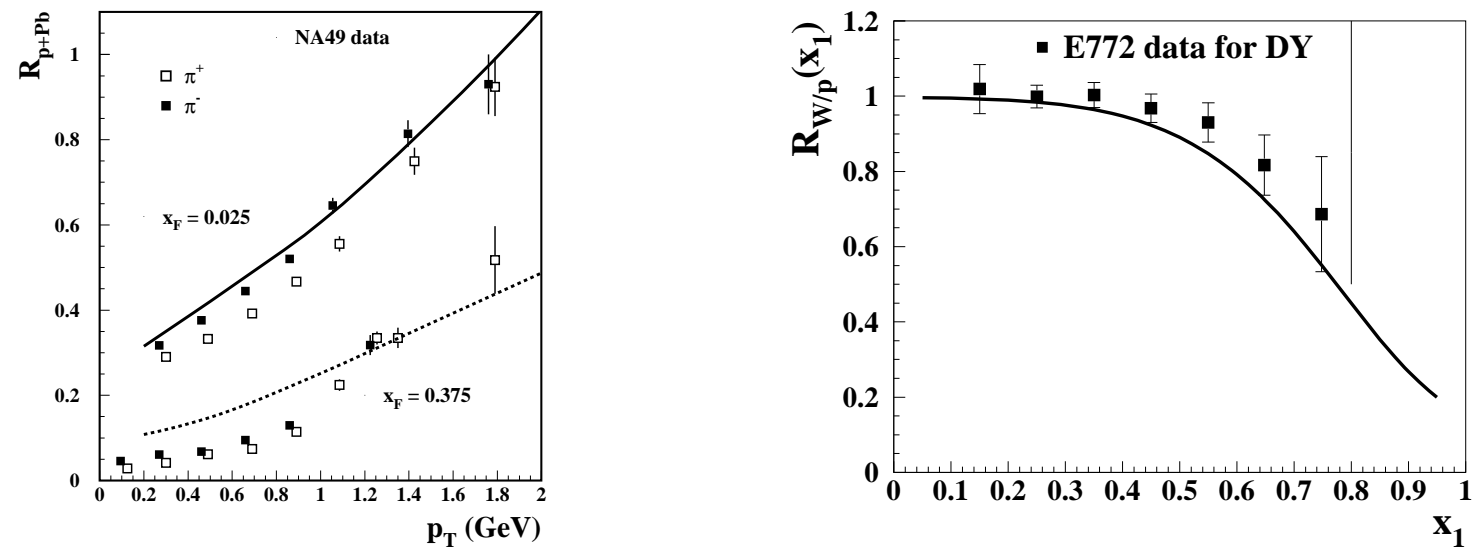

Figure 1: (Left) Ratio, $R_{p+P b}\left(p_{T}\right)$, for $\pi^{ \pm}$production rates in $\mathrm{p}+\mathrm{Pb}$ and $\mathrm{p}+\mathrm{p}$ collisions as function of $p_{T}$ [5] at $E_{l a b}=158 \mathrm{GeV}$ and two fixed $x_{F}=0.025$ and 0.375 vs. NA49 data [f]. (Right) Ratio $R^{D Y}(W / D)$ of Drell-Yan cross sections on W and D [5] vs. E772 data [7] at $E_{l a b}=800 \mathrm{GeV}$ for $6<M<7 \mathrm{GeV}$.

\section{Nuclear suppression at small energies}

Fig. 1 and the left panel of Fig. 2 clearly exhibit the same pattern as that seen at RHIC [1, 9] - a significant rise of suppression with $x_{F}\left(x_{1}\right)$. All those fixed target experiments have too low energy for the onset of coherent effects in gluon radiation since the coherence length, $l_{c} \propto 1 /\left(x_{2} m_{N}\right)$, is shorter than the mean inter-nucleon spacing.

The mechanism of nuclear suppression can be interpreted as a energy dissipation of the projectile hadron and its debris when propagating through the nucleus. As a result, the probability of production of a particle carrying the substantial fraction $x_{F}$ of the initial momentum decreases compared to a free proton target [3, 5 ]. 

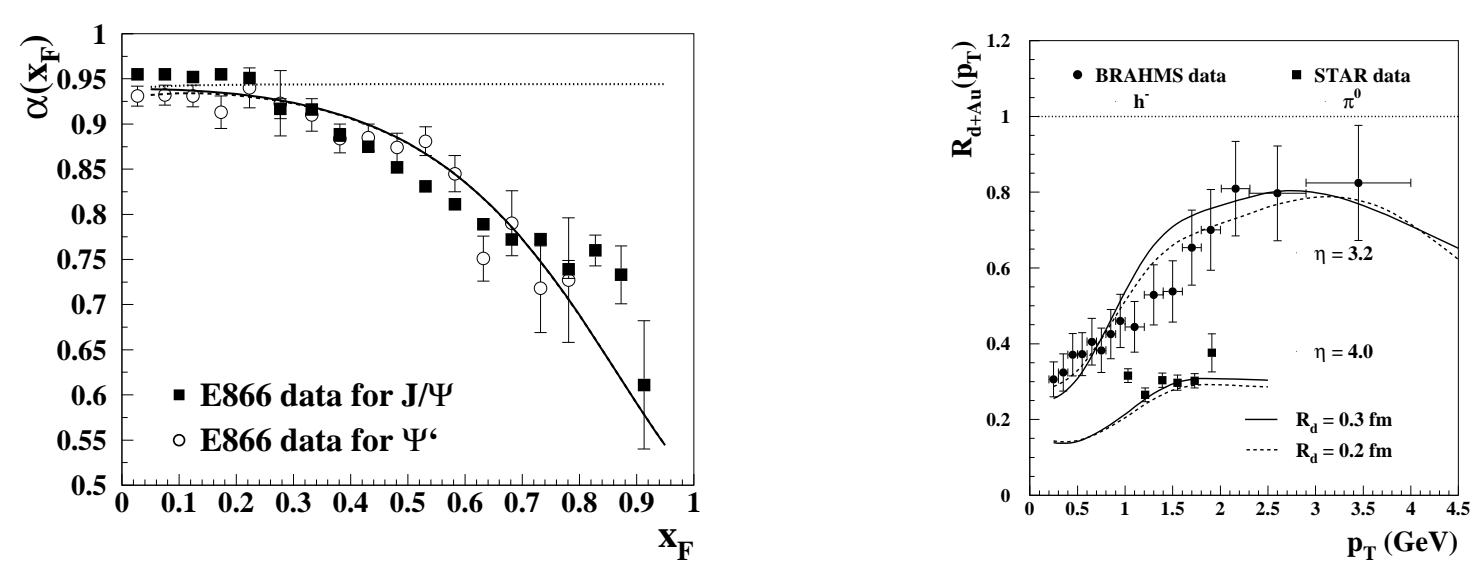

Figure 2: (Left) The exponent describing the A-dependence $\left(\propto A^{\alpha}\right)$ of the nucleus-to-nucleon ratio for the charmonium production as function of $x_{F}$ [5] vs. E866 data [8] at $E_{l a b}=800 \mathrm{GeV}$. (Right) Ratio of $h^{-}$and $\pi^{0}$ production rates in $\mathrm{d}+\mathrm{Au}$ and $\mathrm{p}+\mathrm{p}$ collisions as function of $p_{T}$ at pseudorapidity $\eta=3.2$ and $\eta=4 \mathrm{vs}$. data from the BRAHMS [1] and STAR [9] Collaborations, respectively.

\section{Nuclear suppression of hadrons at RHIC}

The BRAHMS Collaboration [1] reported a significant suppression of $h^{-}$at $\eta=3.2$. Later, however, the STAR Collaboration [9] found much stronger suppression of $\pi^{0}$ at larger $\eta=4$. All these data are consistent with model calculations [3] (see the right panel of Fig. 2) including besides coherent phenomena also corrections for energy conservation. Note, that the onset of coherent effects alone cannot successfully describe a rise of nuclear effects with $y$.

Besides large $x_{F}$ one can approach the kinematic limit increasing $x_{T}=2 p_{T} / \sqrt{s}$. In this case again the energy conservation constraints cause a nuclear suppression. The $\mathrm{d}+\mathrm{A}$ to $\mathrm{p}+\mathrm{p}$ ratio was predicted correctly including also the Cronin effect [10] at medium-high $p_{T}$. Assuming QCD factorization one expects that this ratio should approach one at large $p_{T}$ (with small corrections for isotopic effects). However, corrections for energy conservation lead to a considerable suppression [5], which seems to be confirmed by data presented on the left panel of Fig. 3.
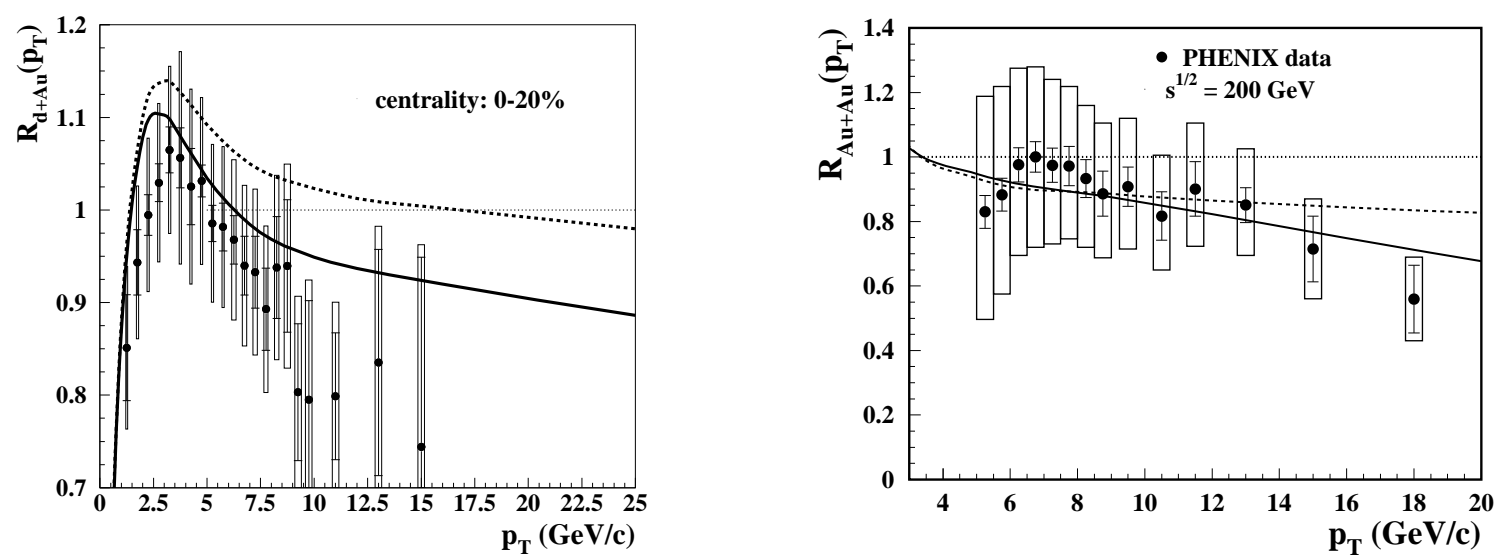

Figure 3: (Left) Nuclear modification factor for $\pi^{0}$ produced in $\mathrm{d}+\mathrm{Au}$ collisions at a centrality range $0-20 \%$ vs. PHENIX data [1]]. (Right) Nuclear modification factor for direct photon production in Au+Au collisions at a centrality range $0-10 \%$ vs. PHENIX data [12]. Solid and dashed lines represent calculations [5] with and without corrections for energy conservation, respectively. 


\section{Direct photons at central rapidity}

Prompt photon production in a hard reaction should not be accompanied with any final state interaction, either energy loss, or absorption. Therefore, besides the Cronin enhancement at mediumhigh $p_{T}$ and small isotopic corrections at larger $p_{T}$ we should not expect any nuclear effects.

Unexpectedly, the PHENIX data [12] exhibit a significant suppression at large $p_{T}$ as is shown in the right panel of Fig. 3. If corrections for energy conservation are not included model calculations [3, 5] depicted by the dashed line give a value $R_{A u+A u} \rightarrow 0.8$ in accord with isotopic effects. Otherwise we predict strong nuclear effects at $p_{T}>10 \mathrm{GeV}$ as is demonstrated by the solid line.

\section{Summary}

Interpretation of a strong nuclear suppression at forward rapidities should be presented with caution. Assuming that only gluon saturation induces the suppression observed at RHIC, one arrives at a small amount of gluons in nuclei breaking down the unitarity bound [13].

Treating the nucleus to be an universal filter for different Fock components of the projectile hadron, one comes to factorization of soft and hard interactions. However, this is not the case at large $x_{F}$ where sharing of energy between the constituents becomes an issue and higher Fock components are resolved better. This effect can be treated as an effective energy loss proportional to initial energy leading so to $x_{F}$ scaling of the suppression. This provides also an explanation for the longstanding puzzle of $J / \Psi$ suppression scaling in $x_{F}$.

Besides large $x_{F} \rightarrow 1$ the kinematic limit can be approached also in transverse momentum increasing $x_{T}$. Similar effects of energy conservation are expected to be manifested. As a result, the Cronin enhancement of particle production at medium-high $p_{T}$ switches to a suppression at larger $p_{T}$. Such an unexpected effect demonstrating a violation of the QCD factorization seems to be confirmed by data for pion production in $\mathrm{d}+\mathrm{Au}$ collisions at RHIC, and even for direct photons. Acknowledgments: This work was supported in part by the Slovak Funding Agency, Grant 2/0092/10 and by Grants VZ MŠMT 6840770039 and LC 07048 (Ministry of Education of the Czech Rep.).

\section{References}

[1] I. Arsene et al. [BRAHMS Collaboration], Phys. Rev. Lett. 93, 242303 (2004).

[2] D. Kharzeev, Y.V. Kovchegov and K. Tuchin, Phys. Lett. B 599, 23 (2004).

[3] B.Z. Kopeliovich et al., Phys. Rev. C 72, 054606 (2005); Nucl. Phys. B 146, 171 (2005); J. Nemchik et al., Phys. Rev. C 78, 025213 (2008); J. Nemchik and M. Šumbera, Nucl. Phys. A 830, 611c (2009).

[4] A.V. Abramovsky, V.N. Gribov and O.V. Kancheli, Yad. Fiz. 18, 595 (1973).

[5] B.Z. Kopeliovich and J. Nemchik, "High-pT paradigms revisited", e-Print: arXiv:1009.1162[hep-ph].

[6] B. Boimska [NA49 Collaboration], Ph.D. Dissertation, Warsaw 2004, CERN-THESIS-2004-035.

[7] D.M. Alde at al. [E772 Collaboration], Phys. Rev. Lett. 64, 2479 (1990).

[8] M.J. Leitch et al. [E866 Collaboration], Phys. Rev. Lett. 84, 3256 (2000).

[9] J. Adams J et al. [STAR Collaboration], Phys. Rev. Lett. 97, 152302 (2006).

[10] B.Z. Kopeliovich, J. Nemchik, A. Schäfer and A.V. Tarasov, Phys. Rev. Lett. 88, 232303 (2002).

[11] S.S. Adler et al. [PHENIX Collaboration], Phys. Rev. Lett. 98, 172302 (2007).

[12] T. Isobe et al. [PHENIX Collaboration], J. Phys. G 34, S1015 (2007).

[13] B.Z. Kopeliovich et al., Phys. Rev. C 79, 064906 (2009). 